\title{
Implicações da pandemia COVID-19 para a segurança alimentar e nutricional no Brasil
}

\author{
Covid-19 pandemic implications for food and nutrition security \\ in Brazil
}

Rita de Cássia Ribeiro-Silva (http://orcid.org/0000-0002-8387-9254) 1,2

Marcos Pereira (https://orcid.org/0000-0003-3766-2502) ${ }^{2,3}$

Tereza Campello (https://orcid.org/0000-0002-9905-9453) ${ }^{4}$

Érica Aragão (https://orcid.org/0000-0002-4903-0556) 2,3

Jane Mary de Medeiros Guimarães (https://orcid.org/0000-0002-9538-2675) 3,5

Andréa JF Ferreira (http://orcid.org/0000-0002-6884-3624) ${ }^{2}$

Maurício Lima Barreto (http://orcid.org/0000-0002-0215-4930) 2,3,6

Sandra Maria Chaves dos Santos (http://orcid.org/0000-0002-4706-0284) ${ }^{1}$
${ }^{1}$ Departamento Ciência da Nutrição, Escola de Nutrição, Universidade Federal da Bahia (UFBA). Av. Araújo Pinho 32, Canela. 40110-150 Salvador BA Brasil.rcrsilva@ufba.br ${ }^{2}$ Rede CoVida Ciência, Informação e Solidariedade. Salvador BA Brasil.

${ }^{3}$ Instituto de Saúde Coletiva, UFBA. Salvador BA Brasil.

${ }^{4}$ University of Nottingham. Nottingham Reino Unido.

${ }^{5}$ Campus Jorge Amado, Universidade Federal do

Sul da Bahia. Itabuna BA Brasil.

${ }^{6}$ Centro de Integração de Dados e Conhecimentos para Saúde, Instituto Gonçalo Moniz, Fiocruz. Salvador BA Brasil.

\begin{abstract}
The emergence of COVID-19 in Brazil further explained the massive discrepancy between different social realities coexisting in the country, rekindling the discussions about food and nutrition security, similarly to what has been happening in other countries facing the same pandemic situation. In this paper, we argue that the risks to hunger and food security in Brazil have been present since 2016 and are now being exacerbated due to the emergence of the COVID-19 epidemic. This situation requires knowing the extent and magnitude of the issue and articulation of measures in the three governmental spheres(federal, municipal and state) to ensure access to adequate and healthy food and reduce the disease's adverse effectson the diet, health, and nutrition among the most vulnerable people. Thus, this work aims to contribute to the debate on the measures to be adopted by governments and society to promote and ensure food and nutrition security and prevent insecurity and the expansion of hunger during and after the social and health crisis created by the pandemic.
\end{abstract}

Key words COVID-19, Pandemics, Food and nutritional security, Public policy
Resumo O surgimento da COVID-19 no Brasil explicitou ainda mais a enorme discrepância entre diferentes realidades sociais que coexistem no país, reacendendo as discussões acerca da segurança alimentar e nutricional, à semelhança do que vem acontecendo em outros países que enfrentam a mesma situação de pandemia. Argumenta-se neste trabalho que os riscos para a Segurança Alimentar e Nutricional (SAN) e a fome dos brasileiros já vinham se apresentando desde 2016, sendo agora aprofundados pela emergência da epidemia da COVID-19, passando a exigir compreensão da extensão e da magnitude dos problemas e articulação de medidas governamentais nas três esferas de gestão (federal, municipal e estadual), que possam assegurar o acesso à alimentação adequada e saudável, com vistas a reduzir os impactos negativos da doença na condição de alimentação, saúde e nutrição dos mais vulneráveis. Assim, este texto pretende contribuir para o debate sobre as medidas a serem adotadas pelos governos e sociedade para promover e garantir a SAN e impedir que a insegurança e a expansão da fome avancem durante e após a crise social e sanitária gerada pela pandemia.

Palavras-chave COVID-19, Pandemias, Segurança alimentar e nutricional, Política pública 


\section{Introdução}

Após décadas de declínio constante, a tendência da fome no mundo, que é refletido pela prevalência da desnutrição, foi revertida em 2015. Nos últimos três anos, as taxas permaneceram praticamente inalteradas em um nível ligeiramente abaixo de $11 \%{ }^{1}$. No entanto, o número de pessoas atingidas pela fome vem aumentando. Como resultado, pouco mais de 821 milhões de pessoas no mundo, ou seja, 1 em cada 9 pessoas, ainda passavam fome em 2018 ${ }^{1}$. Estimativas mais recentes registram que mais de 130 milhões de pessoas podem entrar nesta categoria até o final de $2020^{2}$. Além disso, a pandemia pode levar cerca de 49 milhões de pessoas à extrema pobreza em $2020^{3}$, ressaltando o imenso desafio de atingir a meta do Fome Zero até $2030^{1}$.

Observa-se, ainda, que a fome está aumentando em quase todas as sub-regiões da África, tendo a prevalência de subnutrição atingido prevalência de $22,8 \%$ na África Subsaariana e, em menor grau, na América Latina e no Sudoeste Asiático em $2018^{1}$. Também mais de dois bilhões de pessoas, principalmente em países de baixa e média renda, não têm acesso regular a alimentos seguros, nutritivos e suficientes. Mas o acesso irregular também é um desafio para os países de alta renda, incluindo $8 \%$ da população na América do Norte e na Europa ${ }^{1}$.

No Brasil, a desnutrição alcançou até $5,2 \mathrm{mi}$ lhões de brasileiros no triênio $2015-2017^{1}$. A taxa de conceptos nascidos abaixo do peso se manteve em $8,4 \%^{1}$. Além da desnutrição, o sobrepeso e a obsidade continuam a crescer em todas as regiões, particularmente entre adolescentes e adultos ${ }^{1}$; em algumas delas enfrentam epidemia que combina a obesidade e a desnutrição ${ }^{4}$. A baixa ingestão de micronutrientes como ferro, cálcio e vitaminas $\mathrm{A}$ e $\mathrm{D}$, doenças carenciais conhecidas como "fome oculta" desde os estudos de Josué de Castro nos anos 40, do século passado, permanecem sendo importantes problemas nutricionais no país ${ }^{5}$. Essa condição traz sérias implicações para a saúde e o desenvolvimento físico e cognitivo, com efeitos diretos na qualidade de vida das pessoas; a anemia é um dos problemas nutricionais que representam riscos mais significativos para a saúde, particularmente quando ocorre durante a gravidez. Segundo este mesmo relatório, $27,2 \%$ das mulheres em idade fértil no Brasil (14 a 49 anos) sofriam de anemia ${ }^{1}$.

Este conjunto de dados e informações é relevante neste contexto porque, de uma parte, revelam que a pandemia não poderá ser res- ponsabilizada isoladamente pela severidade que se anuncia na situação de fome, desnutrição e insegurança alimentar e nutricional (ISAN) de todos. Antes, as desigualdades não superadas, $\mathrm{o}$ avanço de políticas neoliberais e o desmonte do sistema que contemplava políticas sociais inclusivas e promotoras da SAN vem se somando para a situação atual, que tende ao agravamento dado os impactos da pandemia. Decerto, a insegurança alimentar nos domicílios brasileiros, seja nos gradientes leve, moderada ou grave, deve tender à maior magnitude, com o advento da pandemia da COVID-19. Assim, o objetivo deste artigo é discutir as repercussões da COVID-19 na situação de segurança alimentar e nutricional e sua interface com políticas de proteção social.

Para tanto, foram analisados documentos oficiais sobre decretos e leis relativas ao enfrentamento da COVID-19 no âmbito estadual e federal. Privilegiou-se as ações voltadas à geração de renda e intervenções direcionadas à garantia da alimentação adequada. O ensaio é delineado em três secções: a primeira abrange a conjuntura das políticas de proteção social, a segunda refere-se às dimensões da segurança alimentar e nutricional e por fim, são apresentadas possíveis estratégias para o enfrentamento da fome e trilhar o caminho da SAN no Brasil.

\section{O desmonte das políticas de proteção social no Brasil}

Ao longo das últimas duas décadas, diversas políticas públicas foram criadas para a saída do Brasil do Mapa da Fome ${ }^{6}$. Uma delas é o Programa Bolsa Família (PBF), um programa de transferência condicional de renda $(\mathrm{CCT})^{7}$. Entre 2004 e 2014, o programa aumentou as despesas de $0,29 \%$ para $0,46 \%$ do Produto Interno Bruto (PIB) anual e a cobertura familiar de 6,6 milhões para 14 milhões ${ }^{8,9}$. Estima-se que o componente de transferência de renda adotado pelo programa tenha sido responsável por $25 \%$ de redução da pobreza extrema e por quase $15 \%$ da redução da pobreza desde $2004^{10}$. Seu efeito de distribuição poderia explicar entre $1 \%$ e 1,5\% da redução anual do coeficiente de Gini no país ${ }^{10}$.

A prevalência de subnutrição reduziu de $11,9 \%$ no período 1999-2001 para menos de $2,5 \%$ no período $2008-2010^{1,5}$. Reconhece, ainda, no período, um conjunto de políticas, de programas e de ações que, mesmo não tendo a mesma cobertura do PBF, foram relevantes para promover a redução da pobreza, da fome e da ISAN, no Brasil'. Contudo, as políticas sociais com impac- 
to na renda, na pobreza, e na SAN dos brasileiros perderam força desde 2016, com a ascensão de Michel Temer à Presidência da República ${ }^{11}$.

Conta-se com o desmonte, iniciado em 2016, do Sistema Nacional de Segurança Alimentar e Nutricional, criado em 2006, especialmente pela redução de recursos e da cobertura de programas estruturantes, como o programa de transferência de renda (Programa Bolsa Família), o Programa de Aquisição de Alimentos da Agricultura Familiar, os Programas de incentivo à agricultura familiar, o Programa Água para todos, e pela extinção do Ministério de Desenvolvimento Agrário, além do ato simbólico de retirar o termo Combate à Fome do nome do Ministério de Desenvolvimento Social, dentre outros. Sem dúvida, a aprovação da emenda constitucional 95, em 2016, que congelou os gastos públicos por 20 anos, integra fortemente este cenário ${ }^{10,12}$.

Neste âmbito, o novo governo, que tomou posse em janeiro de 2019, herdou e aprofundou os péssimos resultados dos indicadores sociais, como o incremento significativo do número de pessoas em situação de extrema pobreza ${ }^{13}$. Registra-se que um dos primeiros atos do governo do presidente Bolsonaro foi a extinção do Conselho Nacional de Segurança Alimentar e Nutricional (CONSEA), uma instância consultiva da Presidência da República com expressiva participação da sociedade civil organizada, por isso mesmo, caixa de ressonância de demandas da sociedade, estratégica na agenda de construção das políticas de Segurança Alimentar e Nutricional (SAN).

A desaceleração do crescimento econômico no país em adição às políticas econômicas de austeridade fiscal adotadas pelo governo tem contribuído, ainda mais, para o desmonte das políticas sociais no Brasil. Este processo de desmonte afetou não só o Sistema Único de Saúde (SUS) e o Sistema Único de Assistência Social (SUAS), bem como o Sistema Nacional de Segurança Alimentar e Nutricional (SISAN), com aprofundamento da situação de pobreza e de vulnerabilidade social vivenciado por muitas famílias brasileiras, indo na contramão do processo da redução da desigualdade social e redistribuição de renda, experimentado no país entre os anos de 2003 e $2014^{12,14}$

Nessa pandemia, famílias e populações, em contextos de vulnerabilidade social, podem apresentar maior vulnerabilidade à Covid-19 por conta da desigualdade social presente, sobretudo, entre pessoas de baixa renda - os desempregados e aqueles na informalidade, que necessitam complementar renda, ainda que estejam em progra- ma temporário de transferência de renda ${ }^{15,16}$. Tais condições de vulnerabilidade podem ser mais danosas nas comunidades formadas por famílias que vivem em único cômodo doméstico e com compartilhamentos de materiais de higiene pessoal. Gera-se, portanto, necessidade de superar os desafios em torno das medidas efetivas para a redução da incidência de COVID-19.

A redução do poder de compra das famílias e a alta nos preços dos alimentos nos últimos anos foram os principais fatores que levaram a retrações importantes no consumo alimentar, particularmente de alimentos nutricionalmente mais saudáveis ${ }^{17,18}$. Tudo isso em meio a ambientes alimentares agressivos, repletos de alimentos ultraprocessados (de fácil acesso e baixo custo), agravando, assim, a situação de ISAN entre os mais vulneráveis ${ }^{19}$. Neste contexto, ressaltar-se que o desmonte das políticas públicas e do CONSEA expõe, na pandemia COVID-19, a omissão do Estado na redução de desigualdades e no enfrentamento da insegurança alimentar e nutricional no Brasil e pode deixar as pessoas/famílias - já expostos às privações alimentares - mais vulneráveis à fome antes do início desse cenário epidemiológico.

\section{Segurança alimentar e nutricional: dimensões no contexto da pandemia COVID-19}

A Lei Orgânica de $2006^{20}$ apresenta um conceito de Segurança Alimentar e Nutricional que consiste na realização do direito de todos ao acesso regular e permanente a alimentos de qualidade, em quantidade suficiente, sem comprometer o acesso a outras necessidades essenciais, tendo, como base, práticas alimentares promotoras de saúde que respeitem a diversidade cultural e que sejam ambiental, cultural, econômica e socialmente sustentáveis ${ }^{20}$. A partir de fevereiro de 2010, a alimentação foi incluída entre os direitos sociais previstos no artigo $6^{\circ}$ da Constituição Federal. Entretanto, o Direito Humano a Alimentação Adequada (DHAA) está distante da realidade de muitas pessoas em todo o mundo ${ }^{12}$ e com a pandemia da COVID-19, os desafios são maiores.

Para tematizar os desafios durante a pandemia, pode-se considerar, em termos gerais, duas dimensões bem definidas: a alimentar e a nutricional. A primeira se refere aos processos de disponibilidade (produção, comercialização e acesso ao alimento); e a segunda diz respeito mais diretamente à escolha, ao preparo e ao consumo alimentar e sua relação com a saúde e com a uti- 
lização biológica do alimento. Cada uma delas é permeada pelos objetivos de garantir alimentos saudáveis, que respeitem a cultura alimentar da população e que tenham sido produzidos de forma sustentável ${ }^{21,22}$. Tais dimensões foram afetadas pela pandemia COVID-19 (Quadro 1).

Na dimensão alimentar, as necessárias medidas preventivas de distanciamento e isolamento social adotadas pela grande maioria dos governos estaduais e municipais, seguindo recomendações do Ministério da Saúde e da Organização Mundial de Saúde (OMS), tendem a gerar comprometimentos particularmente para a população mais vulnerável no que concerne à oferta suficiente de alimentos frescos e minimamente processados, em especial os provenientes da agricultura familiar. Diversos agricultores viram os canais de comercialização serem suspensos, seja pelo fechamento temporário de restaurantes, seja pela paralisação das aquisições pelo Programa Nacional de Alimentação Escolar (PNAE), ou, ainda, em virtude da redução (e, em diversos casos, fechamento) da comercialização nas feiras livres e nos mercados. Outros vivenciam dificuldades de comercialização (de alimentos, produtos, artesanato, mão de obra etc.) em virtude de medidas que têm limitado os deslocamentos intermunicipais ou restringido o transporte público.

Ao lado disso, para muitos grupos da agricultura familiar, aqueles que já viviam em situação de pobreza ou limitações nas condições socioeconômicas, a pandemia da COVID-19 pode significar intensificação da vulnerabilidade social. Esses problemas tendem a ter consequências ainda a serem dimensionadas a médio e longo prazo, para além da crise sanitária, pela quebra das cadeias de produção e comercialização de agricultura familiar (Quadro 1).

Ainda na dimensão alimentar, deve se considerar o acesso físico e econômico aos alimentos, sendo essencial a disponibilidade de alimentos e de renda para a aquisição dos itens que representam o padrão alimentar das famílias. O distanciamento social repercute na dinâmica e na condição socioeconômica de inúmeras famílias brasileiras, em especial daquelas em situação de vulnerabilidade socioeconômica. Em julho de 2019 , chegavam a $41,3 \%$ os brasileiros ocupados no mercado de trabalho informal no Brasil, ou seja, mais de 38 milhões, conforme cálculos do Instituto Brasileiro de Geografia e Estatística $(\mathrm{IBGE})^{23}$. Além da informalidade, também a fragilização dos vínculos empregatícios, que já estava em curso no país devido à crise econômica e às políticas adotadas em nome da austeridade, foi exacerbada pela pandemia. Inúmeras atividades foram interrompidas sem que houvesse medidas suficientes de amparo aos trabalhadores que perderam seus meios de subsistência, portanto, de acesso aos bens e serviços essenciais, dentre eles os alimentos.

Na dimensão nutricional, as precárias condições de vida, incluindo a falta de acesso à água e ao saneamento básico bem como a fragilidade dos sistemas de saúde (sistemas de saúde tensos e sobrecarregados pela pandemia), ao incidirem sobre o estado de saúde do indivíduo, sobretudo entre os mais vulneráveis, podem limitar a utilização biológica dos nutrientes e colocá-los, portanto, em risco de desenvolver a má nutrição (em suas diferentes manifestações, em particular, a desnutrição e as carências de micronutrientes ${ }^{24}$. Avaliação mais recente (2017) do estado nutricional dos beneficiários do programa Bolsa $\mathrm{Fa}$ mília aponta $12,6 \%$ e 5,3\% de menores de 5 anos com déficit de crescimento e desnutrição aguda (déficit de peso para altura), respectivamente ${ }^{25}$.

Por outro lado, alerta para o excesso de peso nesta população $(13,3 \%)$, situação que pode ser agravada, amparada pela falta de consumo de alimentos saudáveis e adequados (especialmente pobre em frutas, legumes e verduras, contribuindo para as inadequações de micronutrientes). Um aumento expressivo do consumo de alimentos ultraprocessados é esperado em virtude do preço, da saciedade que propiciam e da facilidade de acesso nesta crise sanitária.

Assim, possíveis consequências da ISAN incluem (direta ou indiretamente) efeitos negativos sobre a saúde e a qualidade de vida. A situação se agrava quando são considerados grupos sociais que ainda não experimentaram um processo de inclusão mais sólido na sociedade para serem alcançados por políticas públicas, tais como remanescentes de quilombolas, populações indígenas, comunidades tradicionais como povos de terreiro, fundo de pasto, quebradeiras de coco, marisqueiras, pescadores artesanais e população em situação de rua, entre tantos outros povos e grupos que conformam a sociedade brasileira. Cabe ainda considerar os idosos (com dificuldades de acesso físico aos pontos de venda de alimentos), seja em suas casas ou institucionalizados. Portanto, a emergência e a disseminação da pandemia causada pelo vírus SARS-CoV-2 traz mais intensidade aos problemas que já vinham se acumulando no que concerne à SAN de todos, especialmente dos mais vulneráveis em termos sociais, econômicos e sanitários, sinalizando para possível aumento de situações de fome. 
Quadro 1. Repercussões da COVID-19 na Segurança Alimentar e Nutricional no Brasil.

\begin{tabular}{|l|l|}
\hline \multicolumn{1}{|c|}{ Dimensão de SAN } & \multicolumn{1}{|c|}{ Repercussões da pandemia Covid-19 } \\
\hline $\begin{array}{l}\text { Disponibilidade de } \\
\text { alimentos }\end{array}$ & $\begin{array}{l}\text { - Prejuízos na oferta de alimentos in natura da agricultura familiar (AF), especialmente as } \\
\text { frutas e os vegetais; } \\
\text { - Paralisação do PNAE e aquisição de alimentos da AF; } \\
\text { - Fábricas de processamento de alimentos fechadas devido a surtos entre trabalhadores; } \\
\text { - Equipamentos de venda e comercialização de alimentos foram fechados: fechamentos } \\
\text { de feiras livres, restaurantes; } \\
\text { - Limitação de transportes de alimentos; }\end{array}$ \\
\hline $\begin{array}{l}\text { Acesso aos } \\
\text { alimentos }\end{array}$ & $\begin{array}{l}\text { - Redução ou suspensão de renda para os mais vulneráveis- trabalhadores informais; } \\
\text { - Redução de cobertura do BF; }\end{array}$ \\
\hline Consumo & $\begin{array}{l}\text { - Redução do consumo de alimentos in natura; } \\
\text {-Aumento do ganho de peso e/ou transtornos alimentares associados à inatividade física } \\
\text { e ao isolamento social; }\end{array}$ \\
\hline Utilização biológica & $\begin{array}{l}\text {-Redução ou ausência de acesso aos serviços de saúde pode tornar crianças, idosos, } \\
\text { gestantes mais vulneráveis as deficiências nutricionais; } \\
\text {-Pessoas/populações sem acessos regular e permanente à água, saneamento e higiene } \\
\text { adequados estão sob o risco de desenvolver a má-nutrição, em particular, a desnutrição e } \\
\text { as carências de micronutrientes. }\end{array}$ \\
\hline
\end{tabular}

Fonte: Elaboração própria.

\section{Estratégias para aplacar a fome e trilhar o caminho da segurança alimentar e nutricional}

Situações de emergência exigem respostas rápidas. Considerando as diferentes dimensões da SAN, as iniciativas e as políticas para sua garantia devem conter ações articuladas que contemplem, a um só tempo, tanto seu componente alimentar (disponibilidade, produção, comercialização e acesso aos alimentos) como nutricional (relacionado às práticas alimentares e utilização biológica dos alimentos), de forma a corrigir os desvios urgentes, e definir um futuro de maior SAN.

De partida, importa dizer que a população em situação de vulnerabilidade em SAN é exatamente a mesma que só poderá cumprir as medidas de isolamento e distanciamento social se puder contar com renda para ficar em casa. Para responder a esta demanda, o congresso aprovou a Renda Básica Emergencial de R\$ 600 por trabalhador, podendo chegar a $\mathrm{R} \$ 1.200$ por família destinada ao público do Cadastro Único, informais (autônomos ou por conta própria) e Microempreendedores Individuais (MEI) ${ }^{26}$. A medida vale por 3 meses; infelizmente, questões logísticas podem comprometer e retardar a liberação das parcelas aos que mais precisam do benefício. No que se refere aos assalariados, o nível de desemprego tem aumentado e a parcela dos trabalhadores está sendo dispensada colocando em risco a renda no curto prazo, durante a pandemia, e no pós-crise, quando os trabalhadores se verão sem perspectiva. Portanto, o impacto na segurança alimentar de parcela das famílias estará comprometido se não forem construídas estratégias de garantia de emprego e renda para esta parcela da população. Soma-se ainda que parte dos estabelecimentos (pequenos e médios negócios, inclusive os ligados a cadeias de alimentos) foram fechados e depende de medidas para não falir e poder reabrir nos pós-pandemia.

Com as medidas de combate à COVID-19, os agricultores familiares estão passando por dificuldade para escoar a produção e enfrentam prejuízos financeiros ${ }^{27}$. A agricultura familiar responde pela maior parte da produção de alimentos destinados ao consumo interno no Brasil, mas tem vulnerabilidade econômica significativa, decorrente de diferentes fatores como limitação no acesso a equipamentos que aumentam a produtividade do trabalho, distância das cidades, escala da produção, exploração de atravessadores, entre outros mecanismos.

Com a pandemia, a comercialização de alimentos, sobretudo em feiras livres e mercadinhos, poderá ser diretamente afetada, limitando a renda das famílias agricultoras e camponesas. Portanto, medidas emergenciais de suporte à produção de alimentos precisam ser acionadas como a criação de seguro emergencial de renda para a agricultura familiar, linhas de crédito diferenciadas, mecanismos de apoio à comerciali- 
zação, que mitigam eventuais efeitos negativos no equilíbrio econômico e financeiro dos produtores. Infelizmente, agricultores familiares ainda esperam que medidas emergenciais anunciadas pelo governo federal saiam do papel e cheguem ao campo. Promessas como investimentos nos programas de Aquisição de Alimentos (PAA) e Nacional de Alimentação Escolar (PNAE) - para compras de produtos dos agricultores -, além da criação de linhas de créditos especiais, esbarram na falta de regulamentação e de condições para a execução.

No que se refere à comercialização dos produtos da agricultura familiar, é preciso ampliar iniciativas que permitam a entrega de alimentos, sobretudo dos frescos e saudáveis às pessoas com maiores dificuldades em chegar aos pontos de venda; especialmente relevantes para idosos e pessoas em risco. Em geral são recursos de geoprocessamento que permitem identificar locais de produção e comercialização ativos, por tipo de alimentos, de tal forma que consumidores possam seguir consumindo e os produtores possam produzir, comercializar e manter a vida. Decerto um recurso como este é útil na emergência, com os comércios e feiras paralisados, mas também pode ser incorporado ao cotidiano pós-pandemia. E obviamente, promoção de medidas sanitárias que permitam aumentar a segurança alimentar e contribuam para conscientizar da importância do uso de medidas de higiene para reduzir a transmissão do vírus SARS-CoV-2 e proteger trabalhadores e consumidores.

A preocupação com o acesso aos alimentos é grande na crise. $\mathrm{O}$ Brasil tem experiência com programas que incidem e garantem consumo alimentar adequado para grupos relevantes. O Programa Nacional de Alimentação Escolar (PNAE), representa um dos principais meios de garantir alimentação adequada aos escolares brasileiros durante os dias letivos, e um dos mercados mais importantes para geração de fonte de renda para muitos agricultores familiares. $\mathrm{O}$ fechamento das escolas, medida necessária para conter a propagação do vírus, impôs a interrupção desta política.

Infelizmente, $o$ atraso na orientação federal e na coordenação do Sistema Nacional de Educação no que se refere ao PNAE gerou grande discrepância nas iniciativas de governos estaduais e municipais, que tentaram amortecer os impactos da interrupção das aulas e de fornecimento da alimentação escolar emitindo normas e decretos, para garantir que os recursos ou os alimentos chegassem às famílias carentes e em situação de vulnerabilidade.
A entrega de cestas básicas tem superado a transferência direta de renda, na modalidade de cartão/vale alimentação, e depende da escolha das autoridades locais. Os órgãos que optaram pelas cestas básicas apontam a necessidade de uma compra em grande quantidade para atender ao maior número de pessoas atingidas pelo distanciamento social recomendada pelos governos locais. Por outro lado, o cartão/vale alimentação é apontado por aqueles que o adotam como um meio mais ágil para aumentar a renda e garantir a segurança alimentar de famílias que antes contavam com a alimentação escolar para parte dos membros da família, reduzindo os gastos diários com alimentos.

Em Resolução № 2, de 9 de abril de $2020^{28}$, o Ministério da Educação/Fundo Nacional de Desenvolvimento da Educação autoriza a distribuição de gêneros alimentícios adquiridos no âmbito do PNAE às famílias dos estudantes na forma de Kits. O abastecimento das escolas com alimentos produzidos por pequenos agricultores é uma maneira eficiente de fortalecer a agricultura familiar e reduzir a pobreza ao promover o combate à fome. Por outro lado, importa manter ao máximo a oferta de uma alimentação adequada e saudável aos escolares, conforme preconizam as normas do programa depois de anos de luta para alcançar tal definição. No entanto, mais uma vez, o governo federal agiu à revelia dos entes federados, e a medida foi implementada em descompasso com o que já vinha sendo implementado pelos estados e municípios. A escuta aos estados e municípios seria imperativa, já que a parcela federal é a menor parte do recurso na composição da merenda escolar, e que parcela dos entes não conta com alternativas de estoque e reorganização dos produtos para dispor no modelo exigido pela norma federal. Esta descoordenação impediu que parte dos recursos fosse utilizado. Impera que as diferentes realidades do Brasil exigem que as medidas federais levem em conta os limites e os potenciais de cada comunidade, ainda mais em se tratando de alimentos.

Tendo em vista a necessidade de alimentos para a população vulnerável, os restaurantes populares, apesar dos desmontes, têm se organizado e colocado suas estruturas e funcionários para preparar alimento e distribuir para as populações vulneráveis; portanto sendo estratégicos para assegurar a entrega de refeições à população de rua, idosos em situação de pobreza, e trabalhadores informais. Acertadamente, alguns deles distribuindo refeições prontas em bandejas descartáveis. Para evitar aglomerações em filas, o horário de distribuição também tem sido ampliado. 
Outras iniciativas estão sendo criado para prover ajuda humanitária às pessoas mais atingidas pela crise econômico-social causada pela pandemia da COVID-19. As Organizações Não Governamentais (ONGs), igrejas, associações comunitárias e grupos anônimos, têm desempenhado papel fundamental no combate à COVID-19, estando na linha de frente, em especial nas favelas e nos territórios onde o poder público não chega, repassando cestas básicas àqueles atingidos pela pobreza e extrema pobreza (ex: Ação da Cidadania, uma das maiores e mais respeitadas ONGs do país). Em meio à pandemia, a Companhia Nacional de Abastecimento (Conab), anunciou um leilão para aquisição de alimentos das cestas básicas em apoio às pessoas em situação de vulnerabilidade, agravada pela pandemia; medida essa que pode chegar tardiamente frente à emergência da situação $0^{29}$.

Para aumentar o ônus da COVID-19, os ambientes superlotados e sem condições de seguir recomendações de higiene que garantam a qualidade sanitária e o preparo dos alimentos se desenha no Brasil. Não se pode desconsiderar os riscos da transmissão do SARS-CoV-2 por alimentos contaminados caso tenham sido expostos à secreção respiratória de uma pessoa contaminada. Assim, as comunidades onde são constatadas situações de insuficiência e/ou precariedade nos serviços de abastecimento de água, os moradores acabam obrigados a lançar mão de estratégias de sobrevivência que também levam a outras situações de riscos para a proliferação do SARS-CoV- $2^{30}$.

Particularmente em questões relacionadas à nutrição, é o momento de compartilhar preocupações, alertar e, quem sabe, ampliar o campo de ideias e de práticas para reorientar as pessoas no rumo da boa nutrição. O isolamento social impõe importantes mudanças socioculturais, redução da atividade física e alterações nos hábitos alimentares, determinantes consideráveis do estado nutricional. Evidências convincentes mostraram que os hábitos alimentares são afetados também por condições de angústia e distúrbios emocionais, em que níveis elevados são associados à má qualidade da dieta ${ }^{24}$. Além disso, emoções como medo e tristeza estão associadas a menos desejo ou motivação para comer e menor prazer durante a refeição ${ }^{24}$. Portanto, neste momento, importa ser criativo para criar alternativas que respeitem o Guia alimentar dos brasileiros, de 2014, recomendado pelo Ministério da Saúde, e que protejam as pessoas da má nutrição ${ }^{31}$.

Em síntese, enquanto ainda há muito a ser conhecido sobre o COVID-19, a influência dessa pandemia na SAN exigirá do poder público, da iniciativa privada e da população ações alinhadas para o enfrentamento da situação sem desconsiderar a insegurança alimentar nas suas várias dimensões. Nesta perspectiva, recomendações são apresentadas.

\section{Considerações finais e recomendações}

Não se discute que os problemas nutricionais crônicos do Brasil só serão superados de forma definitiva com medidas estruturais que reorganizem os sistemas alimentares de forma a torná-los saudáveis, sustentáveis, estimuladores da produção, geradores de emprego e alavancadores do desenvolvimento. Mas há um quadro real acirrado pela crise sanitária e pelas medidas de distanciamento social que vem sendo aplicadas no Brasil, resultando em impactos econômicos profundos nas condições de renda e emprego. Os brasileiros podem morrer por falta do que comer. Assim, ao desvelar essa situação, urge a necessidade de saídas que coloquem a vida e a dignidade humana no centro das decisões e políticas públicas, salvaguardando os direitos humanos. No caso do DHAA, significa garantir que todas as pessoas, especialmente as mais vulneráveis, tenham acesso a alimentos adequados e saudáveis para poder atender ao clamor de Fiquem em casa.

Assim, o desafio nesse momento é assegurar diferentes mecanismos que contribuam para garantir o DHAA, potencializando diferentes estratégias de abastecimento alimentar. Nesse contexto, o fortalecimento do PAA (notadamente as modalidades de Compra Direta e Compra com Doação Simultânea) e a continuidade da operacionalização do PNAE (ajustado às demandas sanitárias da COVID-19) são algumas das medidas que podem ser rapidamente acionadas. Também políticas de proteção social extraordinárias, como a distribuição da agricultura familiar, obviamente adaptadas aos cuidados sanitários necessários para reduzir o risco de disseminação do SARSCoV-2 são de suma importância como estratégia pontual para mitigar a fome de diversos grupos vulneráveis no Brasil.

Recomenda-se incluir, ainda, iniciativas de educação alimentar e nutricional (por meio de programas educacionais na televisão, virtual ou rádio) que orientem e estimulem a adoção/manutenção de hábitos alimentares saudáveis para toda a família, incluindo o sempre oportuno estímulo ao aleitamento materno exclusivo até os 6 meses como prática nutricional segura e es- 
sencial para crianças menores de 2 anos. Sempre considerando medidas de precaução sanitária. Logicamente, o monitoramento do estado nutricional deve ser considerado. Por fim, reforça-se o fortalecimento do sistema de vigilância e de monitoramento da disseminação do vírus, com vistas a se definirem estratégias alinhadas para o enfrentamento da COVID-19.

\section{Colaboradores}

RC Ribeiro-Silva trabalhou na concepção do texto, nas análises dos dados e na redação. M Pereira, T Campello, AJF Ferreira e SMC Santos trabalharam nas análises dos dados e na redação do texto. E Aragão, JMM Guimarães, ML Barreto trabalharam na revisão crítica do trabalho. Todos os autores aprovaram a versão final do manuscrito.

\section{Agradecimentos}

Grupo de evidências sobre os impactos sociais e econômicos (Rede CoVida) 


\section{Referências}

1. Food and Agriculture Organization of the United Nations (FAO), International Fund for Agricultural Development (IFAD), United Nations Children's Fund (UNICEF), World Food Programme (WFP), World Health Organization (WHO). The State of Food Security and Nutrition in the World: Safeguarding against economic slowdowns and downturns. Rome: FAO; 2019.

2. United Nations Children's Fund (UNICEF). The Impact of COVID-19 on Food Security and Nutrition [Internet]. [cited 2020 Jun 19]. Available from: https:// data.unicef.org/resources/jme-report-2020/

3. World Food Programme (WFP). HungerMap [Internet]. [cited 2020 Jun 19]. Available from: https://hungermap.wfp.org/

4. Uzêda JCO, Ribeiro-Silva RDC, Silva NDJ, Fiaccone RL, Malta DC, Ortelan N, Barrato ML. Factors associated with the double burden of malnutrition among adolescents, National Adolescent School-Based Health Survey (PENSE 2009 and 2015). PLoS ONE 2019; 14(6):e0218566.

5. Vasconcelos FDAG. Josué de castro e a geografia da fome no Brasil. Cad Saude Publica 2008; 24(11):27102717

6. Comisión Económica para América Latina y el Caribe (CEPAL). Panorama Social da América Latina [Internet]. [cited 2020 Jun 19]. Available from: www.cepal. org/es/suscripciones

7. Campello T, Falção T, Costa PV. Brasil Sin Miseria [Internet]. 2015 [cited 2020 May 25]. Available from: http://www.mds.gov.br/webarquivos/publicacao/ brasil_sem_miseria/livro_o_brasil_sem_miseria/ BSM-espanhol.pdf

8. Food and Agriculture Organization of the United Nations (FAO), International Fund for Agricultural Development (IFAD), World Health Organization (WHO), World Food Programme (WFP) y United Nations Children's Fund (UNICEF). El estado de la seguridad alimentaria y la nutrición en el mundo 2019. Protegerse frente a la desaceleración y el debilitamiento de la economía. [Internet]. [cited 2020 Jun 19]. Available from: http://www.fao.org/3/ca5162es/ca5162es. pdf

9. Cecchini S. Programas de transferencias condicionadas en América Latina y el Caribe: tendencias de cobertura e inversión [Internet]. 2017 Jun [cited 2020 May 30]. Available from: https://repositorio.cepal.org/handle/11362/41811

10. Souza LEPF, Barros RD, Barreto ML, Katikireddi SV, Hone TV, Sousa RP, Leyland A, Rasella D, Millett CJ, Pescarini J. The potential impact of austerity on attainment of the Sustainable Development Goals in Brazil. BMJ Global Health 2019; 4:e001661.

11. Rossi P, Mello G. Choque recessivo e a maior crise da história: a economia brasileira em marcha à ré. Centro de Estudos de Conjuntura e Política Econômica - IE/ Unicamp [Internet]. [cited 2020 Jun 19]. Available from: https://www.eco.unicamp.br/images/arquivos/ NotaCecon1_Choque_recessivo_2.pdf
12. Santarelli M, Burity V, Basílio e Silva LN, Prates L, Rizzolo A, Rocha NC, Trabuco L. Da democratização ao golpe: avanços e retrocessos na garantia do direito humano à alimentação e à nutrição adequadas no Brasil. Brasília: FIAN Brasil; 2017.

13. Instituto Brasileiro de Geografia e Estatística (IBGE). Extrema pobreza atinge 13,5 milhões de pessoas e chega ao maior nível em 7 anos. Agência de Notícias. IBGE [Internet]. [cited 2020 Jun 19]. Available from: https://agenciadenoticias.ibge.gov.br/agencia-noticias/2012-agencia-de-noticias/noticias/25882-extrema-pobreza-atinge-13-5-milhoes-de-pessoas-echega-ao-maior-nivel-em-7-anos

14. Santarelli M, David G, Burity V, Rocha NC. Informe Dhana 2019: autoritarismo, negação de direitos e fome. Brasília: FIAN; 2019.

15. Bezerra ACV, Silva CEM, Soares FRG, Silva JAM. Fatores associados ao comportamento da população durante o isolamento social na pandemia de COVID-19. Cien Saude Colet 2020; 25(Supl. 1):2411-2421.

16. Ahmed F, Ahmed N, Pissarides C, Stiglitz J. Why inequality could spread COVID-19. Lancet Public Health 2020; 5(5):e240.

17. Comisión Económica para América Latina y el Caribe (Cepal). Panorama Social de América Latina [Internet]. 2018 [cited 2020 Apr 30]. Available from: https:// www.cepal.org/pt-br/publicaciones/44412-panorama-social-america-latina-2018-documento-informativo

18. Brinkman H-J, De Pee S, Sanogo I, Subran L, Bloem MW. High food prices and the global financial crisis have reduced access to nutritious food and worsened nutritional status and health. J Nutr 2010; 140(1):153S-161S.

19. Monteiro CA, Cannon G, Levy RB, Moubarac JC, Louzada MLC, Rauber F, Khandpur N, Cediel G, Neri D, Martinez-Steele E, Baraldi LG, Jaime PC. Ultra-processed foods: What they are and how to identify them. Public Health Nutr 2019; 22(5):936-941

20. Brasil. Lei no 11.346, de 15 de setembro de 2006. Cria o Sistema Nacional de Segurança Alimentar e Nutricional - SISAN com vistas em assegurar o direito humano à alimentação adequada e dá outras providências. Diário Oficial da União 2006; 18 set.

21. Hoddinott J. Technical guides for operationalizing household food security in development projects. Washington: International Food Policy Research Institute; 1999.

22. Maxwell S, R. Frankernberger T. Household Food Security: Concepts, Indicators, Measurements. Techinical Review. New York: Unicef; 1992.

23. Instituto Brasileiro de Geografia e Estatística (IBGE). Síntese de indicadores sociais: uma análise das condições de vida da população brasileira. Rio de Janeiro: IBGE; 2019.

24. Naja F, Hamadeh R. Nutrition amid the COVID-19 pandemic: a multi-level framework for action. Eur J Clin Nutr 2020; 1-5. 
25. Brasil. Vigilância Alimentar e Nutricional - DATASUS [Internet]. [cited 2020 Jun 19]. Available from: https://datasus.saude.gov.br/acesso-a-informacao/ vigilancia-alimentar-e-nutricional/

26. Brasil. Auxílio emergencial: 96 milhões de pessoas solicitaram beneficio pelo aplicativo, explica Onyx - TV Senado [Internet]. [cited 2020 Jun 10]. Available from: https://www12.senado.leg.br/tv/programas/ noticias-1/2020/05/96-milhoes-de-brasileiros-acessaram-aplicativo-para-solicitar-auxilio-emergencial -explica-onyx

27. Borsatto RS, Grigoletto F, Macedo AC. Respostas dos municípios para garantir segurança alimentar e nutricional em tempo de pandemia [Internet]. [cited 2020 Jun 19]. Available from: https://preprints.scielo.org/ index.php/scielo/preprint/view/163/222

28. Brasil. Resolução № 2, de 9 de abril de 2020. Dispõe sobre a execução do Programa Nacional de Alimentação Escolar - PNAE durante o período de estado de calamidade pública, reconhecido pelo Decreto Legislativo $n^{\circ} 6$, de 20 de março de 2020 , e da emergência de saúde pública de importância internacional decorrente do novo coronavírus - Covid-19. Diário Oficial da União 2020; 13 abril.

29. Companhia Nacional de Abastecimento (Conab). Leilão para aquisição de alimentos das cestas básicas é adiado [Internet]. [cited 2020 Jun 19]. Available from: https://www.conab.gov.br/ultimas-noticias/3335-leilao-para-aquisicao-de-alimentos-das-cestas-basicas -anunciadas-pelo-governo-e-adiado

30. Oliveira TC, Abranches MV, Lana RM. (In)Segurança alimentar no contexto da pandemia por SARS-CoV-2. Cad Saude Publica 2020; 36(4):e00055220

31. Brasil. Ministério da Saúde (MS). Guia Alimentar para a População Brasileira. Brasília: MS; 2014.

Artigo apresentado em 24/06/2020

Aprovado em 28/06/2020

Versão final apresentada em 30/06/2020 